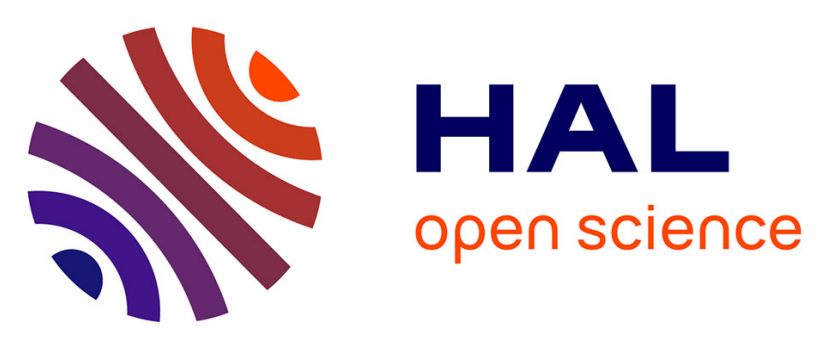

\title{
Persistent contamination of heater-cooler units for extracorporeal circulation cured by chlorhexidine--alcohol in water tanks
}

S. Romano-Bertrand, M. Evrevin, C. Dupont, Jean-Marc Frapier, J.-C. Sinquet, E. Bousquet, B. Albat, E. Jumas-Bilak

\section{To cite this version:}

S. Romano-Bertrand, M. Evrevin, C. Dupont, Jean-Marc Frapier, J.-C. Sinquet, et al.. Persistent contamination of heater-cooler units for extracorporeal circulation cured by chlorhexidine-alcohol in water tanks. Journal of Hospital Infection, 2018, 99 (3), pp.290-294. 10.1016/j.jhin.2018.01.005 . hal-01790459

\section{HAL Id: hal-01790459 \\ https://hal.umontpellier.fr/hal-01790459}

Submitted on 22 Jan 2020

HAL is a multi-disciplinary open access archive for the deposit and dissemination of scientific research documents, whether they are published or not. The documents may come from teaching and research institutions in France or abroad, or from public or private research centers.
L'archive ouverte pluridisciplinaire HAL, est destinée au dépôt et à la diffusion de documents scientifiques de niveau recherche, publiés ou non, émanant des établissements d'enseignement et de recherche français ou étrangers, des laboratoires publics ou privés. 


\section{Persistent contamination of heater-cooler units for extracorporeal circulation cured by chlorhexidine-alcohol in water tanks}

\section{S. Romano-Bertrand ${ }^{a, *}$, M. Evrevin ${ }^{b}$, C. Dupont ${ }^{c}$, J.-M. Frapier ${ }^{d}$, J.-C. Sinquet ${ }^{d}$, E. Bousquet ${ }^{b}, B$. Albat ${ }^{d}, E$. Jumas-Bilak ${ }^{a}$}

a Hydrosciences Montpellier, IRD, CNRS, Univ Montpellier, Département d'Hygiène Hospitalière, CHU Montpellier, Montpellier, France

bépartement d'Hygiène Hospitalière, CHU Montpellier, Montpellier, France

'Hydrosciences Montpellier, IRD, CNRS, Univ Montpellier, Montpellier, France

d PhyMedExp, INSERM, CNRS, Univ Montpellier, Service de Chirurgie Thoracique et Cardiovasculaire, CHU Montpellier, Montpellier, France

\section{Keywords:}

Heater-cooler units Non-tuberculous mycobacteria Stenotrophomonas maltophilia Pseudomonas aeruginosa Chlorhexidine-alcohol

\section{$S U M M A R Y$}

Recently, surgical site infections due to non-tuberculous mycobacteria (NTM) have been linked to heater-cooler unit contamination. The European Centre for Disease Prevention and Control and manufacturers now recommend the use of hydrogen peroxide in filtered water to fill heater-cooler unit tanks. After implementation of these measures in our hospital, heater-cooler units became heavily contaminated by opportunistic waterborne pathogens such as Pseudomonas aeruginosa and Stenotrophomonas maltophilia. No NTM were detected but fast-growing resistant bacteria could impair their detection. The efficiency of hydrogen peroxide and chlorhexidine-alcohol was compared in situ. Chlorhexidine-alcohol treatment stopped waterborne pathogen contamination and NTM were not cultured whereas their detection efficiency was probably improved.

\section{Introduction}

Heater-cooler units (HCUs) used for extracorporeal circulation in cardiac surgery are a recognized bacterial reservoir

\footnotetext{
* Corresponding author. Address: Centre Hospitalier Regional Universitaire de Montpellier Infection Control, 15 Avenue du Doyen Gaston Giraud, Montpellier 34095, France. Tel.: +33411759430.

E-mail address: sara.romano-bertrand@umontpellier.fr (S. RomanoBertrand).
}

for waterborne pathogens such as pseudomonas and legionella [1]. However, the transmission from HCUs and their involvement in surgical site infections (SSIs) have been demonstrated only recently, with worldwide clustered cases of cardiac SSI caused by Mycobacterium chimaera, a non-tuberculous mycobacterium (NTM) originating from HCUs [1,2]. This outbreak led to the provision of a rapid risk assessment by the European Centre for Disease Prevention and Control (ECDC) in April 2015 that recommended the implementation of disinfection practices for the microbiological control of HCUs $[3,4]$. 
Heater-cooler unit manufacturers subsequently recommended adding medical-grade hydrogen peroxide in the filtered water used for filling the HCU tanks; and this unstable solution was to be changed weekly [4]. Additionally, a weekly disinfection of the tank using biocides from a list provided by the manufacturer was recommended. Hydrogen peroxide and other recommended biocides were chosen for their activity against NTM. After implementation of this protocol in our hospital, water in HCU tanks became heavily contaminated by fast-growing waterborne pathogens such as Pseudomonas aeruginosa and Stenotrophomonas maltophilia.

This study aimed to evaluate disinfection protocols by microbiological sampling of water from HCU tanks in our hospital. For this, we compared, in situ, the efficiency of the recommended hydrogen peroxide disinfection method with another protocol using chlorhexidine-alcohol at $0.1 \%$ final concentration.

\section{Methods}

The cardiac surgery ward of the University Hospital of Montpellier used four 3T HCUs (LivaNova, London, UK). The manufacturer recommended the weekly replacement of the hydrogen peroxide, and a full decontamination every two weeks, but this time-consuming protocol quickly turned into an in-house degraded protocol named herein 'hydrogen peroxide protocol': water tanks were decanted every two weeks and filled with filtered (Aquasafe filter; Pall Corporation, Port Washington, New York, USA) tap water containing $100 \mathrm{~mL}$ of medical-grade $3 \%$ hydrogen peroxide, with the addition of $50 \mathrm{~mL}$ of $3 \%$ hydrogen peroxide every week [4]. A full HCU decontamination using Puristeri ${ }^{\circledR} 340$ (Fresenius Medical Care, Bad Homberg, Germany) was performed once a month. A new protocol, named 'chlorhexidine-alcohol protocol', subsequently replaced hydrogen peroxide with $0.5 \%$ chlorhexidine-alcohol, with the same frequencies of replacement and decontamination as during 'hydrogen peroxide protocol'.

The water tanks of the four HCUs were sampled twice a month, prior to disinfection. Half a litre of water was aseptically sampled in a sterile bottle containing sodium thiosulphate (20 mg/L final concentration) (Gosselin, Borre, France). Water was filtered through $0.45 \mu \mathrm{m}$-pore-size cellulose sterile filters (Sartorius, Göttingen, Germany) using a vacuum filter apparatus. Filters were deposited on to plate count agar (PCA) (bioMérieux, Marcy l'Etoile, France) to enumerate the total mesophilic bacteria, and on to cetrimide and MacConkey agar plates (bioMérieux) for the detection of Pseudomonas aeruginosa and Enterobacteriaceae, respectively. Media were incubated for $72 \mathrm{~h}$ at $30^{\circ} \mathrm{C}$. To detect NTM, $50 \mathrm{~mL}$ of water was decontaminated using $4 \% \mathrm{NaOH}$ for 15 min. After neutralization using $\mathrm{pH} 6.8$ phosphate buffer, the suspension was centrifuged at $3000 \mathrm{~g}$ for $30 \mathrm{~min}$. Pellet $(500 \mu \mathrm{L})$ was plated on to blood agar medium and Middlebrook Agar 7H11 medium (bioMérieux). The decontamination step could be avoided if the water sample was free from fast-growing mesophilic bacteria. Media were incubated for 10 days at $30^{\circ} \mathrm{C}$.

All colony morphotypes were identified by matrix-assisted laser desorption/ionization time-of-flight mass spectrometry, using Microflex and MALDI Biotyper database (Bruker, Billerica, MA, USA).

\section{Results}

Table I shows the bacterial inoculum in HCU tanks water and the presence of waterborne pathogens, NTM, and fungi, according to the period of the decontamination protocol. The evolution of the bacterial contamination in the water tank is also displayed in Figure 1. Prior to March 2017, the four HCUs were contaminated by mean bacterial inoculum varying from $7.10 \times 10^{1}$ to $7.4 \times 10^{3}$ colony-forming units (cfu) per $100 \mathrm{~mL}$; and contamination was slightly lower for HCU1 and 2 compared to HCU3 and 4 (Table I, Figure 1). In each HCU, the bacterial inoculum varied widely from 0 to $5.10^{4} \mathrm{cfu} / 100 \mathrm{~mL}$ according to the sample and independent to the date of sampling.

Waterborne opportunistic pathogens such as $P$. aeruginosa and S. maltophilia were detected as well as other nosocomial pathogens (Enterobacter cloacae, Enterococcus faecalis, and Klebsiella pneumoniae) but no NTM were isolated (Table I). It was noteworthy that $P$. aeruginosa and S. maltophilia partially resisted the in-laboratory decontamination step and probably impaired the detection of NTM. Several fungi were also retrieved in the four HCUs: Aspergillus niger, Aspergillus fumigatus, and Fusarium spp. (Table I).

After March 2017, the chlorhexidine-alcohol protocol was tested for filling HCU1 and HCU2 tanks. The hydrogen peroxide protocol continuing for HCU3 and HCU4 were deemed as controls. The use of chlorhexidine-alcohol did not impair the function of HCUs but perfusionists observed the formation of scale deposit in tubing, requiring their monthly replacement. The two control HCUs were still highly contaminated, mainly by S. maltophilia and filamentous fungi (Table I, Figure 1). NTM were not detected but again the microbiological analysis for their detection was complicated by S. maltophilia, which resisted the pre-analytical in-laboratory decontamination step. During October 2017, the chlorhexidine-alcohol protocol was extended to HCUs 3 and 4, permitting suppression of the waterborne pathogens, without the detection of NTM. Hence, the chlorhexidine-alcohol protocol eliminated all growing bacteria, including S. maltophilia and fungi. NTM were not detected during the whole study period.

\section{Discussion}

Prior to April 2015, microbiological control of HCU tank water was not regularly performed in the Hospital University of Montpellier. After an environmental investigation around a cardiac SSI case caused by Mycobacterium wolinskyi during which Mycobacterium chelonae was isolated from an HCU, microbial contamination was regularly analysed as was also recommended by the ECDC rapid risk assessment [5]. During this period, the bacterial load reached $10^{4}$ cfu per $100 \mathrm{~mL}$ and S. maltophilia seems to have been gradually selected. Despite wide variations in load, the persistence of bacterial contamination suggested that bacterial growth and biofilm formation were not impaired by the hydrogen peroxide protocol. By contrast, the chlorhexidine-alcohol protocol eliminated bacterial contamination and did not select NTM as was feared, due to their intrinsic tolerance to chlorhexidine.

Due to the worldwide outbreak of M. chimaera, NTM are considered as a risk factor for cardiac surgery SSIs when present in HCUs. The risk associated with other micro-organisms colonizing HCU tanks appears to be lower. In our hospital, a 
Table I

Follow-up of microbiological contamination of heater-cooler units (HCUs)

\begin{tabular}{lllllll}
\hline Period/protocol & HCU & Water tanks & $\begin{array}{c}\text { Mean inoculum in } \\
\mathrm{cfu} / 100 \mathrm{~mL} \text { (range) }\end{array}$ & NTM & $\begin{array}{c}\text { Pathogenic bacteria } \\
\text { (\% of positive samples) }\end{array}$ & $\begin{array}{c}\text { Fungi } \\
\text { (\% of positive samples) }\end{array}$
\end{tabular}

February 2016 to March 2017

Hydrogen peroxide protocol

$$
\begin{array}{ll}
\text { HCU } 1 \text { Cardioplegy } & 7 \times 10^{1}\left(0-10^{4}\right) \\
& (N=20)
\end{array}
$$

\begin{tabular}{|c|c|c|c|c|}
\hline \multirow[b]{3}{*}{ HCU 2} & Patient & $1.1 \times 10^{2}\left(0-5.10^{4}\right)$ & None & S. maltophilia $(50 \%)$ \\
\hline & \multirow{3}{*}{ Cardioplegy } & $(N=20)$ & & Enterococcus faecalis (5\%) \\
\hline & & $\begin{array}{l}8 \times 10^{2}\left(0-5.10^{4}\right) \\
(N=19)\end{array}$ & \multirow[t]{2}{*}{ None } & $\begin{array}{l}\text { S. maltophilia }(47 \%) \\
\text { P. geruginosa }(5 \%)\end{array}$ \\
\hline \multirow{6}{*}{$\mathrm{HCU} 3$} & & & & Enterobacter cloacae (5\%) \\
\hline & \multirow[t]{3}{*}{ Patient } & $8 \times 10^{3}\left(0-5.10^{4}\right)$ & \multirow[t]{3}{*}{ None } & S. maltophilia $(42 \%)$ \\
\hline & & $(N=19)$ & & P. aeruginosa $(5 \%)$ \\
\hline & & & & Burkholderia cenocepacia (5\%) \\
\hline & Cardioplegy & $\begin{array}{l}5.4 \times 10^{3}\left(0-10^{4}\right) \\
(N=19)\end{array}$ & None & S. maltophilia (74\%) \\
\hline & Patient & $\begin{array}{l}6 \times 10^{3}\left(40-10^{4}\right) \\
(N=19)\end{array}$ & None & S. maltophilia $(68 \%)$ \\
\hline
\end{tabular}

HCU 4 Cardioplegy

\section{$6.3 \times 10^{3}\left(0-10^{4}\right) \quad$ None S. maltophilia $(77 \%)$} $(N=13)$

Patient

$$
\begin{aligned}
& (N=13) \\
& <1(N=6) \\
& <1(N=6)
\end{aligned}
$$$$
7.4 \times 10^{3}\left(0-10^{4}\right)
$$

April to September 2017

Chlorhexidine-alcohol protocol

Hydrogen peroxide protocol

$\mathrm{HCU}$

Cardioplegy

Patient

HCU 2 Cardioplegy $<1(N=6)$

Patient $\quad<1(N=6)$

HCU 3 Cardioplegy $10^{4}\left(10^{4}\right)(N=7)$

Patient

HCU 4 Cardioplegy

$10^{4}\left(10^{4}\right)(N=7)$

$10^{4}\left(10^{4}\right)(N=8)$

Patient

$$
10^{4}\left(10^{4}\right)(N=8)
$$

HCU1

Cardiopleg
Patient

$<1(N=3)$

$<1(N=3)$

HCU2

Patient

HCU3

Cardioplegy

HCU4
Patient

$<1(N=3)$

$<1(N=3)$

$<1(N=3)$

$<1(N=3)$

Cardioplegy $<1(N=3)$
None

Stenotrophomonas

maltophilia (45\%)

Pseudomonas aeruginosa (5\%)

None

S. maltophilia $(69 \%)$

Klebsiella pneumoniae (8\%)

None None

None None

None None

None None

None S. maltophilia (100\%)

None S. maltophilia (86\%)

None S. maltophilia (88\%)

None

S. maltophilia $(75 \%)$

K. pneumoniae (12\%)

$\begin{array}{lll}\text { None None } & \text { None } \\ \text { None None } & \text { None } \\ \text { None None } & \text { None } \\ \text { None None } & \text { None } \\ \text { None None } & \text { None } \\ \text { None None } & \text { None } \\ \text { None None } & \text { None } \\ \text { None None } & \text { None }\end{array}$

Aspergillus niger (5\%)

Fusarium (30\%)

Penicillium (10\%)

Yeast $(10 \%)$

Fusarium (20\%)

Penicillium (15\%)

Fusarium (32\%)

Fusarium (32\%)

Penicillium (11\%)

Yeast (5\%)

Fusarium (42\%)

Penicillium (11\%)

Yeast (11\%)

Aspergillus

fumigatus (5\%)

Fusarium (37\%)

Penicillium (11\%)

Fusarium (23\%)

Penicillium (8\%)

Yeast (15\%)

Fusarium (23\%)

Yeast $(8 \%)$

None

None

None

None

None

None

Fusarium (17\%)

Penicillium (17\%)

Penicillium (17\%)

None

None

None

None

None

cfu, colony-forming units; NTM, non-tuberculous mycobacteria; $N$, number of samples.

retrospective review of patient case notes showed that no cardiac SSIs caused by $P$. aeruginosa or S. maltophilia occurred in this period (data not shown). Cardiac surgery SSI cases caused by $S$. maltophilia, $P$. aeruginosa or mould reported in the literature have not been linked to HCU contamination; but this potential source of infection was not specifically investigated in these reports $[6,7]$. Importantly, reservoirs of multidrugresistant, bacterial pathogens are not acceptable within a surgical theatre and should be eliminated by a suitable protocol. Moreover, the absence or low load of rapidly growing bacteria avoids mycobacterial culture media contamination, allowing the detection of slow-growing NTM. In this situation, the inlaboratory decontamination of the sample that can affect NTM viability is not required as described for NTM detection in reprocessed bronchoscopes $[8,9]$. In fact, we did not find NTM in our study and we cannot exclude that the decontamination step used to eradicate rapidly growing bacteria was also decontaminating the water samples of mycobacteria [10].

In practice, the manufacturer's recommendation for HCU tank decontamination, i.e. the hydrogen peroxide protocol, 


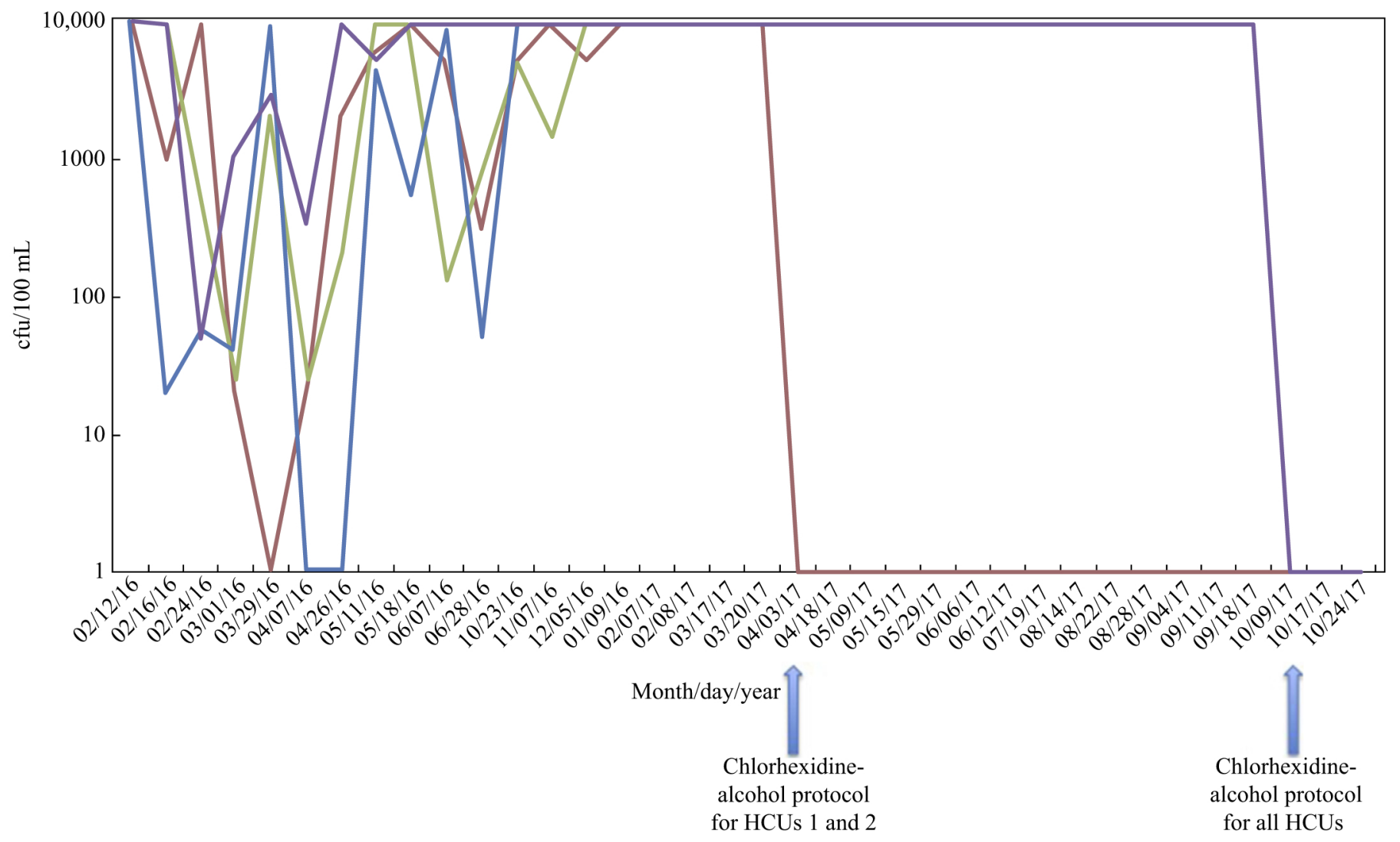

Figure 1. Evolution curves of water tank contamination of heater-cooler units (HCUs). cfu, colony-forming units.

is time-consuming and deviations can occur, due to the workload within cardiac surgery teams. Most reports indicate that change of hydrogen peroxide solution is performed less frequently than recommended [1]. In our hospital, the hydrogen peroxide protocol was followed as scrupulously as possible, considering its cumbersomeness; yet we failed to obtain acceptable microbiological quality for water in HCUs. Other authors have tried successive close cycles of decontamination to improve the decontamination and have concluded that the microbial load in the HCU had not been totally removed, and that the high frequency of decontamination may have led to damage to copper components [11].

In our hospital, the infection control team, in agreement with the cardiac surgery staff and HCU manufacturer, now recommend the use of chlorhexidine-alcohol for HCU decontamination. The tap water used for filling tanks is submitted for antimicrobial filtering and its quality is assessed four times a year. The water in HCU tanks is sampled twice a month, and is subjected to microbiological analysis, including specific search of NTM, with and without in-laboratory decontamination of the sample, thereby ensuring better detection of NTM. The main aspect to monitor while using this new protocol of disinfection is scale formation in the tubing, which needs replacing as soon as scale deposit is observed.

\section{Conflict of interest statement None declared.}

\section{Funding sources}

None.

\section{Acknowledgements}

We thank the perfusionists from the Cardiothoracic Surgery Department and the technicians of the laboratory of hospital microbial ecology for their help in performing tests and analyses of HCU water samples.

\section{References}

[1] Walker J, Moore G, Collins S, Parks S, Garvey MI, Lamagni T, et al. Microbiological problems and biofilms associated with Mycobacterium chimaera in heater-cooler units used for cardiopulmonary bypass. J Hosp Infect 2017;96:209-20.

[2] Williamson D, Howden B, Stinear T. Mycobacterium chimaera spread from heating and cooling units in heart surgery. N Engl J Med 2017;376:600-2.

[3] Haller S, Höller C, Jacobshagen A, Hamouda O, Abu Sin M, Monnet DL, et al. Contamination during production of heater-cooler units by Mycobacterium chimaera potential cause for invasive cardiovascular infections: results of an outbreak investigation in Germany, April 2015 to February 2016. Euro Surveill Bull 2016;21.

[4] Sorin Group. Cardiac surgery mycobacterium risks. Disinfection and cleaning of Sorin heater cooler devices. 2015. Available at: www. livanova.sorin.com/file/download-4303.action [last accessed January 2018].

[5] Dupont C, Terru D, Aguilhon S, Frapier J-M, Paquis M-P, Morquin D, et al. Source-case investigation of Mycobacterium wolinskyi cardiac surgical site infection. J Hosp Infect 2016;93:235-9.

[6] Si D, Rajmokan M, Lakhan P, Marquess J, Coulter C, Paterson D. Surgical site infections following coronary artery bypass graft procedures: 10 years of surveillance data. BMC Infect Dis 2014;14:318. 
[7] Vazquez R, Vazquez-Guillamet MC, Suarez J, Mooney J, Montoya JG, Dhillon GS. Invasive mold infections in lung and heart-lung transplant recipients: Stanford University experience. Transpl Infect Dis 2015;17:259-66.

[8] Radomski N, Cambau E, Moulin L, Haenn S, Moilleron R, Lucas FS. Comparison of culture methods for isolation of nontuberculous mycobacteria from surface waters. Appl Environ Microbiol 2010;76:3514-20.

[9] Falkinham JO. Hospital water filters as a source of Mycobacterium avium complex. J Med Microbiol 2010;59:1198-202.
[10] Schreiber PW, Köhler N, Cervera R, Hasse B, Sax H, Keller PM. Detection limit of Mycobacterium chimaera in water samples for monitoring medical device safety: insights from a pilot experimental series. J Hosp Infect 2017 Nov 21. https:// doi.org/10.1016/j.jhin.2017.11.007 [Epub ahead of print].

[11] Garvey MI, Ashford R, Bradley CW, Bradley CR, Martin TA, Walker J, et al. Decontamination of heater-cooler units associated with contamination by atypical mycobacteria. J Hosp Infect 2016;93:229-34. 